\title{
UN ESTUDIO TRANSVERSAL RETROSPECTIVO SOBRE PROLONGACIÓN Y ABANDONO DE ESTUDIOS UNI- VERSITARIOS
}

\section{[A retrospective transversal study of enlargement and college dropout]}

por

\begin{tabular}{c|c|c}
$\underline{\text { Article record }}$ & $\begin{array}{c}\text { Lidia Cabrera (dcabrera@ull.es) } \\
\text { José Tomás Bethencourt Benítez } \\
\text { Mbout authors }\end{array}$ & $\begin{array}{c}\text { Ficha del artículo } \\
\text { HTML format }\end{array}$ \\
\hline Pedro Álvarez Pérez & $\underline{\text { Sobre los autores }}$ \\
\hline Formato HTML
\end{tabular}

Abstract
At present leaving and prolonging studies constitutes a
serious problem at the university level. This report pre-
sents the results of a retrospective transversal study
which purpose was to identify risk factors related with
dropout. The methodology design used can be for help
in the building of predictive models about dropout.
Keywords
Dropout studies; university orientation; disability
learning; retrospective study; transversal study

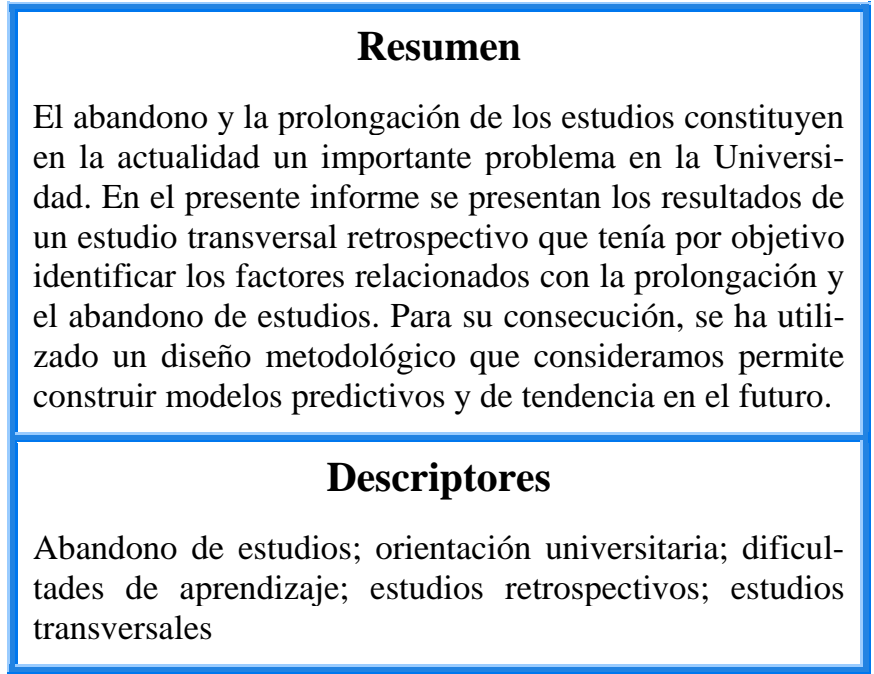

\section{Introducción}

Los estudios longitudinales se han erigido en el tiempo como la estrategia metodológica más adecuada para el análisis del desarrollo de eventos, naturales y sociales, en un perio- do de tiempo suficientemente largo. Ejemplo de ello son los Estudios Evolutivos, en los que el factor determinante es el cambio producido por el paso del tiempo, o los Estudios Epidemiológicos aplicados al estudio del origen y distribución o frecuencia de fenó- 
menos en grupos sociales, y los factores que influyen sobre la ocurrencia y variación de esta distribución (Colimon, 1990). Estos estudios, trascienden los objetivos de identificación y descripción de eventos para ampliarse a los siguientes: conocer la naturaleza de los problemas o fenómenos objeto de estudio, y la variación de los mismos según el tiempo y lugar, a través de índices de prevalencia e incidencia; conocer el perfil o características del fenómeno; determinar las causas; y predecir el desarrollo, tanto a nivel comunitario como individual. En la actualidad, la utilización del tiempo en investigación nos lleva a plantearnos cinco tipos de investigaciones longitudinales: estudios simultáneos, de intervención, de panel, de seguimiento y de tendencias (Keeves, 1997)

Sin embargo, el desarrollo de estudios de estas características entraña innumerables dificultades, no siempre posibles de superar, por el coste de tiempo, esfuerzo y dinero, así como por la imposibilidad en muchas ocasiones de contar con personas colaboradoras en la investigación a las que se les pueda hacer este seguimiento en el tiempo, o porque el fenómeno ya ha sucedido y no se puede reconstruir. Por otro lado, las necesidades prácticas exigen a los investigadores disponer de datos de forma inmediata, con el fin de poder dar respuesta con similar inmediatez. Todo ello sin contar con la presión hacia la rentabilidad y productividad a la que está sometido el profesorado universitario desde las distintas administraciones. Estas circunstancias exigen que fenómenos que, por su propia naturaleza, necesitan una observación y análisis a lo largo del tiempo sean abordados con otras estrategias de menor coste y máximo beneficio, sobre todo en favor de la toma de decisiones prácticas para la prevención e intervención para la mejora. Un ejemplo de ello es la investigación del abandono y prolongación de los estudios universitarios. Los altos porcentajes de incidencia, conocidos a través de las distintas administraciones públicas, exigen analizar el fenómeno para determinar su naturaleza, a través de la iden- tificación de factores asociados, diseñar acciones y estrategias que frenen su aparición, así como poder predecir el futuro riesgo de abandono, que sirva de referencia tanto a los departamentos de orientación de secundaria y servicios de orientación universitarios, como a los propios estudiantes antes de tomar decisiones sobre sus elecciones vocacionales.

Un estudio longitudinal de este fenómeno, en su forma clásica, hubiera exigido iniciar un seguimiento a través de un estudio descriptivo de una promoción o cohorte de estudiantes. La cohorte ${ }^{[1]}$ la constituye un grupo de personas que comparten una misma condición o característica; en este caso alumnado que inicia estudios universitarios, con la finalidad de averiguar cuántos no finalizan y los factores determinantes de esa circunstancia. A través de ese seguimiento obtendríamos datos de interés que nos permitirían plantear hipótesis explicativas de las causas del abandono y la prolongación de los estudios. Pero el tiempo medio de realización de una carrera universitaria en España es de cinco años, por lo que tendríamos que esperar todo ese tiempo para responder a una necesidad y demanda social que exige acciones inmediatas destinadas a frenar el fenómeno. Por ello, decidimos poner en práctica una alternativa metodológica en la que combinamos una modalidad de estudio transversal o de corte, de larga tradición en otras disciplinas, como la medicina (Aibar y col., 1999) o la psicopatología (González de Rivera, 1993), pero de un modo restrospectivo.

Un estudio transversal constituye el estudio de un evento en un momento dado, superando así la limitación del factor tiempo. En este caso la unidad de tiempo viene determinada sólo por las exigencias de las condiciones del estudio, es decir por el necesario para recoger y analizar los datos, pues un estudio transversal o de corte equivale, como dice Cólimon (1990), a tomar una fotografía. Para poder analizar entonces la incidencia del paso del tiempo, se opta por "analizar simultáneamente los componentes de una muestra 
en la que figuren sujetos de diferentes edades de forma que cubran el arco de tiempo propuesto en el estudio" (Mateo, 2004). Obviamente, en nuestro caso, no resultaba útil a nuestros objetivos de investigación escoger cinco grupos representativos de los distintos niveles de estudio, por lo que hubo que hacerlo de un modo retrospectivo, es decir, analizando el fenómeno o efecto ya producido, buscando hacia atrás posibles explicaciones a las situaciones presentes. Esta estrategia metodológica nos permitió hacer el seguimiento del efecto o la influencia de las variables estudiadas en el abandono y prolongación de los estudios, reconstruyendo el pasado a partir de los datos recogidos en el momento del estudio, después de producido el efecto (después de pasados varios años desde el inicio de estudios universitarios). Las variables o eventos se registraron por tanto de forma simultánea, al amparo del recuerdo de los encuestados, a quienes instamos a reflexionar sobre la presencia en el desarrollo de la carrera de las circunstancias objeto de análisis como factores causales. En el desarrollo del estudio, mostraremos las cuestiones relacionadas con el procedimiento.

Nuestro interés por el estudio del abandono y la prolongación de la permanencia del alumnado en la universidad proviene del incremento de casos registrados en el conjunto del Estado Español, lo que ha provocado que se encienda una luz de alarma en las universidades. Según datos estadísticos del Ministerio de Educación, esta realidad es una constante en todas las comunidades autónomas, donde en algunas de ellas el abandono de los estudios sin finalizar roza el $40 \%$. Concebimos el abandono como las situaciones en las que el alumnado deja la Universidad o la carrera iniciada sin finalizarla. Por prolongación de la permanencia entendemos el número de años adicionales a lo prescrito en el plan de estudios que un estudiante emplea para obtener el título de la carrera que estudia, ya sea de ciclo corto o largo.
Este fenómeno, sin embargo, no es ajeno a otras zonas del planeta, si bien es cierto que las causas identificadas difieren de unas zonas o países a otros. Según la UNESCO (2004), a través de la Asociación Internacional de Universidades (IAU), ésta es una constante en unos 180 países. Por otro lado, la literatura pone de manifiesto esta realidad, así lo muestran los estudios de Albert y Toharia (2000); Orazem (2000); Callejo (2001); Escandell y col. (2002); Last y Fulbrook (2003); Ryan y Glenn (2003); Orfield (2004) y Feldman (2005), entre otros, así como su escasa atención en España.

Los estudios revisados identifican una gran constelación de causas relacionadas con una amplia variedad de situaciones, y que van desde el abandono por la mala formación previa (Zubieta y Susinos, 1986), el origen social (Latiesa, 1992), la baja autoestima (Masjoan, 1989), la escasa integración académica y social (Tinto, 1975) u otras características psicológicas como incapacidad para demorar las recompensas o superar obstáculos (Landry, 2003), por el agotamiento de las convocatorias de exámenes (Escandell y Marrero, 1999), la elección inadecuada de estudios (Corominas, 2001), hasta dificultades en las estrategias de aprendizaje (Ryan y Glenn, 2003) o características familiares o circunstancias de vida (Sinclair y Dale, 2000). Por todo ello, actualmente, el abandono se ha convertido en un tema preocupante y de gran interés en todos los niveles. Así lo demuestra, entre otros, el estudio de Van Ours y Ridder (2003), que analizan la relación entre la supervisión del profesorado y las tasas de abandono en el tercer ciclo; o los realizados en torno a la secundaria obligatoria y postobligatoria (López Rupérez, 2004, 2005), que compara las tasas de abandono del sistema español con el resto de socios comunitarios y profundiza en los factores determinantes del mismo.

Estos datos avalan la necesidad de llevar a cabo estudios a través de los cuales se identifiquen con mayor claridad las causas que 
están detrás del abandono y prolongación de la permanencia del alumnado en la enseñanza superior, con el fin de poner en práctica acciones encaminadas a hacer frente a la incidencia que este problema tiene en la institución. La deserción del alumnado universitario de sus estudios es uno de los múltiples indicadores de la calidad del sistema educativo, que pone de relieve la existencia de serios fallos en los procesos de orientación, transición, adaptación y promoción del estudiantado.

Abordar el estudio de este fenómeno exige ampliar el análisis desde el alumnado a la comunidad, pues con el abandono de los estudios son muchos los implicados, como factores desencadenantes, y muchos los afectados. El primero es el alumnado, por el daño y sufrimiento psicológico que le produce esa experiencia de fracaso personal, que se extiende a su familia. La institución universitaria no queda exenta de frustración, pues los fracasos del alumnado remueven los cimientos institucionales, al no poder rendir cuentas socialmente desde los parámetros públicos de calidad. Finalmente, el tercer actor del fenómeno es el Estado, por el desequilibrio que supone para el sistema de educación superior, que afecta al conjunto social, por los enormes costos económicos que produce sin alcanzar los objetivos formativos poblacionales esperados.

Conjugar el estudio de una constelación tan amplia de variables, con la urgente necesidad de obtener resultados concluyentes de ayuda a la intervención, es un reto complejo pero no imposible de superar. Estudios precedentes, nacionales como el de Corominas (2001) o internacionales como el de Lipka (2004) en EE.UU., hicieron posible la formulación de hipótesis etiológicas. Al amparo de estos resultados previos, nosotros nos atrevimos a poner en práctica la metodología transversal de corte retrospectivo. Definimos la cohorte en el pasado, estableciendo un espacio temporal suficiente para finalizar la titulación elegida y recoger los datos, tanto del efecto como de los factores relacionados, en un momento determinado o corto periodo de tiempo.

\subsection{Los estudios transversales o de corte de tipo retrospectivo}

Este método se refiere al estudio de uno o más eventos de distribución poblacional en un momento dado, para averiguar el índice de prevalencia en un área o institución. Esta secuencia cronológica les sitúa frente a los estudios longitudinales, que hacen un seguimiento del factor de riesgo en el tiempo. Sin embargo, la finalidad de los estudios transversales también puede hacer el seguimiento del efecto que produce en el tiempo el factor de riesgo o variable causal, a través de la reconstrucción hacia el pasado, de un modo retrospectivo, es decir reconstruyendo el pasado a partir de los datos recogidos en el presente. Por ello, en los estudios transversales se puede identificar no sólo el porcentaje del fenómeno o evento (número de casos), sino también identificar factores relacionados para establecer asociaciones de causalidad (un factor de riesgo y un efecto). Cuando el corte se refiere a la distribución de frecuencia del fenómeno objeto de estudio, se trataría de un estudio de prevalencia, y equivaldría a una especie de censo que puede repetirse en el tiempo. Cuando lo que pretendemos es establecer relaciones de asociación, uno es presumiblemente el factor de riesgo y, por tanto, es obligado que esté presente antes de que suceda el evento, erigiéndose como factor determinante del efecto o efectos. En nuestro estudio transversal se revisaron todos los eventos de forma simultánea, con una mirada hacia atrás. El suponer que unos anteceden al otro en este binomio, se hizo de acuerdo a datos provenientes de otros estudios descriptivos previos, así como de conclusiones a partir de la observación cotidiana de la realidad objeto de análisis.

Nos encontramos, por tanto, ante una modalidad de diseño transversal retrospectivo, donde se trata de ver la influencia o relación de las variables independientes (factores de- 
terminantes del abandono de estudios) sobre la dependiente (el abandono de estudios), pero sin crear diseño experimental, es decir, sin manipular las variables independientes. Esta modalidad de diseño es adecuada a circunstancias en las que las variables independientes no se pueden manipular, porque ya se ha producido el efecto como en nuestro caso, o porque hay cuestiones que éticamente tampoco es posible provocar, como también sucede con el abandono de estudios; en este caso, procede por tanto reconstruir las causas del efecto ya producido, bien para minimizar su efecto ofreciendo una reorientación al alumnado que deserta, pero, sobre todo, para impedir que suceda en otros casos en el futuro. Este tipo de estudios son denominados dentro de la literatura de metodología de la investigación ex post facto (después del hecho), o comparativo causal. En estas situaciones se identifican las condiciones que ya han tenido lugar, para averiguar la relación de estas circunstancias sobre el comportamiento posterior.

Dentro de los estudios transversales, sin embargo, nos encontramos con otra modalidad, atendiendo al momento en el que se realiza el estudio respecto a la determinación del efecto producido por el factor de riesgo: los estudios prospectivos (Salthouse $\mathrm{y}$ Schroeder, 2004). Esta modalidad de investigación trata igualmente de reconstruir los hechos, pero invirtiendo el orden en la búsqueda de información; es decir, estudiamos el comportamiento de personas con una condición o característica determinada (variable independiente) para ver en el tiempo su posible influencia o relación con la variable dependiente que estamos estudiando, por lo que se mide antes la variable independiente, y no las dos a la vez como en la modalidad restrospectiva. En la investigación retrospectiva, además, estimamos primero el valor de la variable dependiente (abandono), para buscar después hacia atrás las posibles explicaciones de la misma, es decir, estimamos la relación de ésta con las posibles variables independientes. La presencia de una covariación sistemática entre los dos tipos de variables será la clave que nos permitirá determinar las posibles causas explicativas. Para ello, y para ofrecer mayores garantías científicas a nuestra investigación (validez interna y externa), utilizaremos un diseño retrospectivo de grupo único.

El diseño de grupo único, representa, junto con el diseño simple y con el diseño de grupo cuasi control, la tercera modalidad de los diseños retrospectivos. Con el primero estudiaríamos las relaciones de las variables dependientes e independientes en un mismo grupo que comparten el mismo valor en la variable dependiente, es decir, cumple una condición dada, pero como esta condición (abandona estudios) tiene el mismo valor para todos, apenas se pueden estudiar covariaciones. El segundo diseño nos permite establecer relaciones en dos grupos independientes, uno que cumple la condición de la variable dependiente y otro que no cumpla esa condición (alumnado que abandona, alumnado que no abandona). Estos grupos son elegidos por el investigador de forma intencional, con lo que se garantiza la ocurrencia del fenómeno que se quiere observar (un grupo que abandona y otro que no abandona), pero no se sabe qué valores de las potenciales variables independientes van a aparecer en la muestra; por otro lado, no pueden ser generalizables los resultados por la limitación e intencionalidad con la que han sido elegidos los dos grupos.

Con el grupo único (representativo de una población natural) se puede aumentar el número de variables independientes que se utilizan a la vez. También se aumenta la muestra considerablemente $\mathrm{y}$, por tanto, la generalización de datos es más posible. Es más, en este caso, si trabajamos con muestras lo suficientemente representativas de la población, tendremos casos que recojan todos los valores posibles de las variables implicadas (recordemos que en los diseños complejos el investigador decide qué variables dependientes y elige los grupos en función de esa con- 
dición). En este tipo de diseños, los valores de ambas variables, dependientes e independientes, vienen ya dados, sin embargo, no sabemos los valores potenciales de las variables independientes. Para que el rango de posibles valores esté bien representado, se hace necesario aumentar lo más posible el tamaño y la representatividad de la muestra.

Nuestro objetivo último es construir un modelo explicativo que nos permita predecir el riesgo de abandono de estudios. Por ello, nos pareció más adecuado realizar un estudio que no sólo gozara de suficiente validez externa, sino que nos permitiera obtener datos para realizar comparaciones entre grupos que presentaran valores distintos en la variable dependiente. En nuestro caso, a partir de ello identificamos tres grupos independientes: abandonan, prolongan, y terminan. En el apartado referido al método, ilustraremos con mayor detalle el procedimiento seguido tanto en el diseño como en el desarrollo de la investigación.

\subsection{Identificación de variables deter-} minantes del abandono de los estudios universitarios

Son diversos los intentos realizados para construir modelos teóricos sobre el fenómeno del abandono de los estudios universitarios. Todos ellos comparten unas características comunes, y centran el análisis básicamente en tres grupos de variables: el alumnado, el profesorado y la institución. Sin embargo, los autores conceden desigual peso a cada uno de ellos. Para Tinto (1975, 1993), por ejemplo, aunque cada estudiante que accede a los estudios superiores viene con sus propios intereses, expectativas e intenciones, lo que determina básicamente su decisión de cambiar o de continuar su proceso formativo es el nivel de integración social y académica que logre en la institución universitaria. En este caso, se entendería por integración social el grado de sintonía y entendimiento que alcanza con los miembros de la comunidad universitaria, especialmente compañeros y profesores. El estudiante se sentirá más integrado en la medida en que sus capacidades le permitan dar respuestas satisfactorias al nivel de exigencia que plantee la institución. A mayor congruencia entre exigencia de la tarea y capacidad de respuesta efectiva a los problemas que plantee el sistema, mayores posibilidades de que el alumnado continúe sus estudios. Sin embargo, no siempre se da esta correlación positiva, por lo que en muchos casos dicha falta de correspondencia deriva en situaciones de fracaso académico, de abandono o cambio de estudios. Para Bean (1990), sin embargo, la integración social y académica depende de las conductas de afrontamiento positivo (acercamiento) o de afrontamiento negativo (evitación), que inciden directamente en la intención (actitud) de dejar o de continuar los estudios, con lo que delega todas las responsabilidades hacia el alumnado.

Junto a éste, otros factores del alumnado identificados, tanto de naturaleza psicológica como educativa, han sido la falta de capacidades o habilidades para hacer frente a las demandas de los estudios universitarios, conocimientos previos no adecuados, actitudes inapropiadas hacia el aprendizaje, la no correspondencia entre las expectativas del alumnado y las características de la titulación elegida, inadaptación a la estructura y organización académica, estilos de aprendizaje no acordes con la titulación cursada, etc. (Kirton, 2000; Wasserman, 2001; Landry, 2003; Gonzalez y col, 2004, entre otros).

En relación con los factores del profesorado cabe destacar las deficiencias pedagógicas (poca claridad en la presentación de la materia; no promover la reflexión ni los juicios críticos; escaso dominio de la materia enseñada; falta de vocación docente), la falta de atención individualizada al estudiantado y escasa dedicación hacia sus tareas profesionales, como han puesto de manifiesto los estudiantes encuestados por Escandell, Marrero y Castro (2002) de la Universidad de las Palmas de Gran Canaria, o el realizado por Van Ours y Ridder (2003) en Holanda. 
Finalmente, entre los factores de la organización académica nos encontramos con ausencia de objetivos claramente definidos por parte de la institución (Universidad, Centros, Departamentos), falta de coordinación tanto horizontal como vertical entre el profesorado de una misma titulación y el sistema de acceso a los estudios, restricción en la oferta de determinadas carreras, etc. Thomas (2002), desarrolla una investigación con un estudio de casos en una universidad inglesa, y concluye que tanto en el acceso a la universidad como en la finalización de los estudios tiene mucha responsabilidad la interacción entre la institución y otros factores externos de carácter social, como grupo social al que se pertenece, otros apoyos sociales acordes con las diferentes necesidades, o currícula institucionales.

Levy-Garboua (1986) propone un modelo economicista con el que intenta explicar que el éxito o el fracaso en el proceso de formación universitaria depende de dos factores: el esfuerzo y las capacidades iniciales del candidato por un lado, y los recursos humanos y materiales que pone a disposición la institución por otro. La eficacia de esa producción, dice Levy-Garboua (1986), puede garantizarse si la institución conoce las capacidades que poseen los estudiantes que comienzan la formación universitaria, lleva a cabo un seguimiento detallado de los mismos y adecua sus estructuras y recursos a sus características. Esta escasa relación entre coste, incluido esfuerzo personal, y beneficio se ha puesto de manifiesto en muchos estudios posteriores como el de Root y col. (2003).

Todos estos datos nos muestran como el abandono de los estudios universitarios es un fenómeno de naturaleza multicausal, como así lo demuestra Corominas (2001), que en un mismo estudio encuentra carencias en la calidad de la enseñanza, déficits en el potencial de aprendizaje del alumno, dudas sobre la elección pertinente de los estudios y otros factores circunstanciales como financieros, compatibilidad con ocupación laboral, etc.
Cualquiera que sea el origen, lo que sí es evidente es que las elevadas tasas de abandono o la prolongación del tiempo establecido para concluir la carrera constituyen actualmente uno de los graves problemas al que se debe buscar solución si se pretende mejorar la calidad de la enseñanza superior. Estas motivaciones fueron las que nos indujeron a llevar a cabo el estudio que presentamos a continuación.

\section{Método}

En este apartado describiremos el procedimiento metodológico utilizado. Tanto el método, un estudio de corte transversal retrospectivo de grupo único, como el diseño, lo presentamos como una aportación metodológica en investigación en educación, dada su escasa tradición y por las matizaciones introducidas en nuestro estudio. Este estudio, además, constituye la primera observación temporal de las variables objeto de estudio, que pasará a constituir un intervalo dentro de una investigación más amplia de series temporales para la predicción o tendencia del abandono de estudios universitarios.

\section{Objetivos}

- Determinar el índice de prevalencia (número de casos) de abandono y prolongación de estudios universitarios.

- Identificar factores asociados a la probabilidad de abandono y prolongación de los estudios universitarios.

- Determinar el grado de influencia de los factores de riesgo identificados en el abandono o prolongación de los estudios universitarios.

- Averiguar qué hacen los estudiantes después de abandonar una titulación.

\section{Diseño e hipótesis}

Después de analizar los resultados de trabajos de otros colegas, así como las distintas realidades económicas y sociales que envuelven a la universidad española, nuestras hipótesis de trabajo fueron las siguientes: 
- Los índices de abandono y prolongación de estudios protagonizados por nuestros estudiantes son superiores a los de cualquier distribución poblacional normal, en la actualidad.

- Los factores asociados al abandono y prolongación de los estudios son de naturaleza multicausal, y están relacionados con las características psicológicas, vitales y educativas del alumnado, en unos casos, y a la ineficacia docente e institucional, en otros.

- La presencia de ciertas características psicoeducativas del alumnado universitario (poco dominio en estrategias de aprendizaje, baja capacidad de persistencia para alcanzar sus metas, poca satisfacción con la carrera, escasa participación en el aula, etc.), supone un alto riesgo de abandono de los estudios elegidos sin finalizar.

El diseño del estudio al amparo de un procedimiento metodológico retrospectivo de grupo único, exige estudiar el total, o un extracto muestral, de una cohorte temporal elegida. En un segundo momento, después de determinar los valores de la variable dependiente (en nuestro caso fue alumnado que abandona, prolonga y finaliza los estudios), el grupo único se divide atendiendo a esos valores, a fin de establecer relaciones comparativas intergrupos con las variables independientes. En este estudio el grupo lo compuso toda la promoción de estudiantes matriculados en tres titulaciones (Universidad de La Laguna), dos de ciclo largo (matemáticas y pedagogía) y una de ciclo corto (trabajo social), pertenecientes a tres grupos vocacionales distintos: psicopedagógico, científicotecnológico y sociojurídico. Se decidió tomar como referencia las promociones que iniciaron sus estudios en los cursos académicos 1996/97 (licenciaturas) y 1997/98 (diplomaturas) respectivamente. Los datos fueron recogidos en el curso académico 2002/03, por lo que se tuvo en cuenta un intervalo de tiempo suficiente (más años de los exigidos por el plan de estudios) para terminar los estudios en los que se habían matriculado y la recogida de datos.

Las variables objeto de estudio, unas como factores causales y otras como efecto, fueron las siguientes:

- Variable dependiente o criterio. Se presenta como una variable cualitativa con tres niveles: abandono, prolongación y finalización de estudios. Es esta la condición sobre la base de la cual seleccionaremos posteriormente los grupos comparativos.

- Variables independientes o factores determinantes. Se presentan todas como variables cuantitativas, con dos indicadores de respuesta: presencia y grado de influencia. Estas, en un principio, se agrupan en torno a cinco factores: estrategias y herramientas de trabajo intelectual; características psicoeducativas del alumnado; características bio-socio-económicas del alumnado; características de la titulación cursada; características del profesorado. En el apartado de resultados se puede ver la relación de cada una de ellas.

\section{Población y muestra}

La población estaba compuesta por 82 estudiantes de trabajo social, 110 estudiantes de matemáticas y 222 estudiantes de pedagogía. Los datos que disponíamos para contactar con estos estudiantes eran los teléfonos de las fichas del profesorado que les dio clase en primero. Esta circunstancia dificultó identificar una muestra a través de un muestreo aleatorio al azar, por cambios de teléfono y de domicilio. Decidimos, por tanto, abordar toda la población (al tratarse de una cantidad relativamente pequeña) y conseguir entrevistar al mayor número de alumnado. La muestra definitiva quedó constituida por 163 estudiantes (el 39\% de la población objeto de estudio), de los cuales 31 estudiantes eran hombres (19\%) y 130 mujeres (79'8\%). Este desequilibrio entre hombres y mujeres representa las características de la población inicial, con un gran porcentaje de mujeres, sobre todo en las titulaciones de pedagogía y 
trabajo social, ocupadas por chicas en un $95 \%$.

\section{Instrumentos de recogida de informa- ción}

Para la recogida de información se elaboró un cuestionario para administrar telefónicamente. El procedimiento de construcción fue el siguiente:

a) Se hizo una revisión bibliográfica de estudios teóricos sobre los estudios universitarios y resultados de investigaciones similares realizados por otros autores. A partir de los mismos se identificaron todos los indicadores relacionados con las variables objeto de estudio incluidas en nuestras hipótesis: causas de abandono y prolongación de estudios universitarios. Dichos indicadores se agruparon por categorías de análisis (factores), a partir de los cuales se elaboró un primer modelo de cuestionario.

b) Se sometió el mismo a la valoración de jueces con la colaboración de un grupo de expertos: profesorado universitario que impartía clases en las tres titulaciones objeto de estudio. Los jueces eliminaron algunos ítems e identificaron otros indicadores que, por su experiencia, consideraban que influían en el abandono y prolongación de los estudios de sus alumnos y alumnas.

c) Una vez rediseñado el cuestionario a partir de las indicaciones de los jueces, se pasó a una muestra piloto, con la intención de identificar errores de construcción de los ítems y problemas de comprensión.

Después de las correcciones oportunas, el cuestionario quedó estructurado con información referida a:

- Datos de identificación personal.

- Datos sociofamiliares.

- Datos académicos: situación académica actual, modalidad de acceso a los estudios matriculados, características de la elección, en caso de abandono qué hizo posteriormente.
- Grado de posesión y nivel de influencia en la situación actual (terminación de los estudios, prolongación de los estudios o abandono) de las siguientes características: estrategias y herramientas de trabajo intelectual; características psicoeducativas del alumnado; características bio-socioeconómicas del alumnado; características de la titulación cursada y características del profesorado.

\section{Resultados}

\subsection{Situación Académica del alumna- do encuestado}

Del alumnado encuestado el 85,3\% (139) era la primera vez que estaba en la Universidad y por tanto la primera titulación universitaria que iniciaba. El resto, el 14,7\% (24), había iniciado antes otra titulación por lo que ya contaba con experiencias anteriores de abandono de estudios universitarios.

Una vez pasados 5 cursos académicos para los licenciados y cuatro para los diplomados, teóricamente el $100 \%$ de los encuestados debía haber finalizado los estudios iniciados en el curso académico 1996/97 (licenciados) y 1997/98 (diplomados). Sin embargo, el $66 \%$ del alumnado no había terminado sus estudios en el tiempo prescrito. La situación del total del grupo encuestado era la siguiente:

- 54 alumnos había terminado sus estudios (33,1\%).

- 63 alumnos seguían cursando dichos estudios (38,7\%).

- 46 alumnos habían abandonado esos estudios (28,2\%).

En el subgrupo que abandonó los estudios encontramos que el $66 \%$ se había matriculado en otra titulación en la misma Universidad (ULL); el 4,2\% se había ido a otra Universidad; el 21,2\% había iniciado otros estudios no universitarios; y el 8,5\% buscó empleo y trabajaba. Por tanto, un 30\% del alumnado abandona la universidad tras fra- 
Cabrera, L., Bethencourt, J. T., González Afonso, M. y Álvarez Pérez, P. (2006). Un estudio transversal retrospectivo sobre prolongación y abandono de estudios universitarios. RELIEVE, v. 12, n. 1, p. 105-127. http://www.uv.es/RELIEVE/v12n1/RELIEVEv12n1_1.htm

casar en el intento de obtener estudios superiores en una titulación.
Por titulaciones, la situación académica del alumnado la podemos ver en la tabla siguiente:

Tabla 1. Situación del alumnado respecto a los estudios iniciados

\begin{tabular}{|l|l|l|l|}
\cline { 2 - 4 } \multicolumn{1}{c|}{} & TERMINAN & PROLONGAN & ABANDONAN \\
\hline PEDAGOGÍA & $24,4 \%(20)$ & $54,9 \%(45)$ & $20,7 \%(17)$ \\
\hline MATEMÁTICAS & $0 \%$ & $22,9 \%(8)$ & $77,1 \%(27)$ \\
\hline TRABAJO SOCIAL & $73,9 \%(34)$ & $19,6 \%(9)$ & $6,5 \%(3)$ \\
\hline
\end{tabular}

Una variable importante para explicar el éxito en los estudios es la elección vocacional. Por ello, analizamos si la titulación cursada fue la elegida en primer lugar o en la que obtuvieron plaza tras ser rechazados en otras titulaciones preferentes. Los resultados nos indicaron que al 37\% de los estudiantes les hubiera gustado hacer otra titulación en la que no obtuvieron plaza. Sin embargo, tras correlacionar ambas variables encontramos que no existía relación entre cursar estudios elegidos en primer lugar y la situación académica del alumnado. Esta circunstancia pudo deberse también a que el alumnado antes de hacer la preinscripción descarta de antemano aquellas carreras para las que no tiene posibilidades de acceso (bien por sus escasas capacidades o por las bajas notas que posee para acceder a la universidad), de tal forma que la variable preinscripción consideramos que no es un criterio adecuado para determinar su grado de preferencia. Por otro lado, dado que la mayoría del alumnado que abandona la titulación inicia otros estudios en la misma universidad, nos indica que por una causa u otra, como el desconocimiento de la carrera, no eligieron la titulación adecuada a sus características educativas y vocacionales.

\subsection{Determinantes de la situación aca- démica}

Independientemente de las relaciones que a priori pretendimos establecer en nuestras hipótesis de partida, y que posteriormente analizaremos, preguntamos directamente a los encuestados cuáles fueron según ellos las causas o factores que determinaron o influ- yeron para que se produjera la situación académica que tenían en el momento de la encuesta: de finalización de estudios, de prolongación o abandono. Las razones según ellos fueron las siguientes:

a) Alumnado que finaliza los estudios a tiempo: en este grupo no se encuentra ningún estudiante de matemáticas, $\mathrm{y}$, en general, no se observan diferencias entre los otros dos grupos. Estos estudiantes consideran que terminaron su carrera en el tiempo porque: se organizaban bien, eran constantes en el estudio, y tenían interés y esfuerzo personal $(44,4 \%)$; les gustaba lo que estudiaban (40\%); tenían ganas de tener un título, trabajar u otras expectativas de futuro $(31,4 \%)$; les motivaba la carrera (29); por las características de la carrera, accesible, duración corta y el ambiente (profesorado y el grupo) (20,3\%); por cumplir las metas propuestas y empeño o deseo por terminar (12,9\%); era lo que habían elegido y tenían muy claro lo que querían (11\%). De forma muy aislada se apuntaron otras razones como presión exterior, ilusión y entusiasmo por estudiar, y factores económicos y de desplazamiento. En este grupo que ya había finalizado sus estudios encontramos que un $62,9 \%$ trabajaba (no sabemos si en trabajos relacionados con su titulación u otros), un 12,9\% estaba en paro, y un 20,3\% seguía estudiando.

b) Alumnado que prolonga sus estudios:La mayor parte del alumnado que prolongó pertenecía a la titulación de pedagogía (73,6\%) siguiéndole trabajo social $(14,9 \%)$ y matemáticas $(11,4 \%)$. En este 
grupo, a pesar de que hay unas características vitales comunes a todos los subgrupos, sí se observaron diferencias. En general, el alumnado de trabajo social asoció la prolongación a cuestiones vitales personales, como muerte o enfermedad de un familiar. El de pedagogía a la desmotivación por la carrera (estructura, no les gustaba, no fue lo que eligieron, incierto panorama laboral, etc.), y a cuestiones relacionadas con la falta de esfuerzo, porque no tenían prisa por acabar. Finalmente, para los de matemáticas las razones más importantes fueron la exigencia de la carrera y la dificultad de los estudios, imposible de superar (no sólo por dificultad sino por cantidad de materia) en menos tiempo.

En conjunto, como en el caso anterior, las razones específicas causantes atribuidas a la prolongación de los estudios fueron: problemas económicos y laborales (incompatibilidad) (41,4\%); características de la carrera (estructura, metodología, cambio de plan e incompatibilidades, no cumplió las expectativas, etc.) (32,2\%); falta de motivación por la carrera y de expectativas laborales $(27,5 \%)$; gran exigencia de la titulación y del profesorado (26,3\%); problemas personales (22,5\%); dificultades con el profesorado y la administración (incomprensión, falta de motivación) (21\%); muerte o enfermedad de familiares $(8,2 \%) ; \mathrm{y}$, finalmente, algunas cuestiones más puntuales e individuales fueron: incompatibilidad con otra titulación, dejadez, no acceder a la carrera que quería, tomárselo de forma muy relajada, inmadurez intelectual, no tomárselo en serio, no tener claro si era esa la titulación deseada, inadaptación, no trabajar bien en grupo, escasos conocimientos previos.

c) Alumnado que abandona sus estudios: La mayor parte del alumnado que abandonó sus estudios pertenecía a la titulación de matemáticas. Sin embargo, todo el alumnado que abandonó indicó causas comunes: desmotivación y falta de ilusión por la carrera, bien porque no resultó ser lo que esperaban (más en trabajo social y pedagogía), o porque era muy difícil (más en el caso de matemáticas). Otras razones más específicas fueron: porque no les gustaba la carrera, no era lo que esperaban, decepción con los contenidos, o demasiada teoría (sobre todo en matemáticas) (45,8\%); alto nivel de exigencia $\mathrm{y}$, por tanto, mal rendimiento y falta de capacidades (39,5\%); siempre quisieron hacer otra titulación (22,9\%), sobre todo en el caso de pedagogía, carrera en la que entran para acceder a segundos ciclos (psicopedagogía, periodismo); falta de motivación por las escasas salidas laborales $(14,5 \%)$. Otras razones muy aisladas, casi unipersonales, fueron: incompatibilidad con trabajo; no gustarle la facultad ni el ambiente; poco tiempo disponible para el estudio; no sentirse valorado ni tenido en cuenta por el profesorado, con relaciones frías y distantes; falta de recursos económicos; problemas personales; falta de concentración y madurez; poca seriedad de la carrera, muy floja y con muchas asignaturas de relleno; falta de colaboración del alumnado; mala evaluación.

\subsection{Razones/Motivos que influyeron en la elección de los estudios}

Las circunstancias que influyeron o determinaron la elección de los estudios, consideramos que forman parte de la constelación de variables explicativas del abandono académico; por ello, las tuvimos en cuenta en nuestro estudio. El análisis global de los datos confirmó que la elección de los estudios estuvo basada fundamentalmente, y por orden de preferencia, en: la preferencia o gusto por la carrera $(75,5 \%)$, seguida por la posesión de habilidades y aptitudes para esa titulación $(68,1 \%)$, y por el deseo de poseer un título (56,5\%).

El resto de las variables analizadas tuvieron una influencia menos relevante en la elección de la carrera. Así, tenemos: calidad de la formación ofrecida en la carrera (34,3\%); no existir límite de plazas para el acceso (31,9\%); no conseguir plaza en la 
Cabrera, L., Bethencourt, J. T., González Afonso, M. y Álvarez Pérez, P. (2006). Un estudio transversal retrospectivo sobre prolongación y abandono de estudios universitarios. RELIEVE, v. 12, n. 1, p. 105-127. http://www.uv.es/RELIEVE/v12n1/RELIEVEv12n1_1.htm

titulación deseada (31,9\%); salidas laborales de la carrera $(21,5 \%)$; consejos de otras personas (20,2\%); facilidad de los estudios $(15,9 \%)$; duración de la carrera $(14,8 \%)$; otros (14,8\%); falta de recursos económicos para estudiar otra carrera fuera de la ULL $(12,3 \%)$; prestigio social de la carrera $(8,5 \%)$.

Los resultados son los mismos al comparar las respuestas en los tres grupos de la investigación (terminan, prolongan y abandonan), no encontrando diferencias significativas entre las razones que les llevaron a elegir los estudios y la situación académica actual, excepto en la variable no conseguir plaza en la titulación que deseaba donde sí existieron diferencias significativas (chi cuadrado = 0,000). Elegir la carrera actual por no conse- guir plaza en la titulación deseada fue la situación más frecuente en el grupo que posteriormente abandonó sus estudios.

\subsection{Estrategias y herramientas de tra- bajo intelectual}

Preguntamos al alumnado sobre el uso o puesta en práctica de algunas herramientas y estrategias de trabajo intelectual, y, posteriormente, le solicitamos que indicara en qué medida creía que ello había influido en su situación actual (finalizar, prolongar, abandonar). Los resultados se muestran en las tablas 2 y 3 . En la tabla 2 los porcentajes hacen referencia al uso, y en la tabla 3 los porcentajes indican la importancia o influencia del uso en sus situaciones ${ }^{[2]}$.

Tabla 2. Percepción del grado de posesión de estrategias y herramientas de trabajo intelectual.

\begin{tabular}{|l|c|c|c|c|}
\cline { 2 - 5 } \multicolumn{1}{c|}{} & TERMINAN & PROLONGAN & ABANDONAN & Chi $^{2}$ \\
\hline Mucho tiempo de estudio & $90,8 \%$ & $74,2 \%$ & $51,1 \%$ & 0,000 \\
\hline Actividades de formación complementaria & $74,1 \%$ & $35,5 \%$ & $6,4 \%$ & 0,000 \\
\hline Uso de técnicas de estudio & $37 \%$ & $32,2 \%$ & $17,1 \%$ & 0,020 \\
\hline Calidad de las técnicas de estudio empleadas & $87,0 \%$ & $90,3 \%$ & $59,6 \%$ & 0,002 \\
\hline Asistencia regular a clase & $96,3 \%$ & $80,7 \%$ & $89,4 \%$ & 0,000 \\
\hline Asistencia a tutorías & $38,9 \%$ & $43,6 \%$ & $23,4 \%$ & 0,007 \\
\hline Trabajo en grupo & $100 \%$ & $87,1 \%$ & $36,2 \%$ & 0,000 \\
\hline Uso de bibliografía & $88,9 \%$ & $82,3 \%$ & $51,1 \%$ & 0,000 \\
\hline
\end{tabular}

Tabla 3. Valoración del grado de influencia de las estrategias y herramientas de trabajo intelectual en su situación actual.

\begin{tabular}{|l|c|c|c|c|}
\cline { 2 - 4 } \multicolumn{1}{l|}{} & TERMINAN & PROLONGAN & ABANDONAN & Chi $^{2}$ \\
\hline Mucho tiempo de estudio & $92,5 \%$ & $74,4 \%$ & $59,5 \%$ & 0,000 \\
\hline Actividades de formación complementaria & $61,2 \%$ & $53,2 \%$ & $19,2 \%$ & 0,000 \\
\hline Uso de técnicas de estudio & $68,5 \%$ & $61,9 \%$ & $40,5 \%$ & 0,002 \\
\hline Calidad de las técnicas de estudio empleadas & $68,5 \%$ & $72,6 \%$ & $38,3 \%$ & 0,003 \\
\hline Asistencia regular a clase & $94,4 \%$ & $70,9 \%$ & $31,9 \%$ & 0,000 \\
\hline Asistencia a tutorías & $42,6 \%$ & $51,6 \%$ & $29,8 \%$ & 0,019 \\
\hline Trabajo en grupo & $90,8 \%$ & $69,4 \%$ & $29,8 \%$ & 0,000 \\
\hline Uso de bibliografía & $87,1 \%$ & $77,4 \%$ & $23,4 \%$ & 0,000 \\
\hline
\end{tabular}


Como podemos ver, los porcentajes son altos en todas las variables de este apartado en los tres grupos. Destacó, sin embargo, como fue disminuyendo de forma constante tanto la presencia como la influencia de todas las estrategias de trabajo en los tres grupos, siendo de mayor influencia en el grupo que termina y de menor en el que abandona.

El porcentaje de personas que dedicó mucho tiempo a estudiar fue mayor en el grupo que terminó, a la vez que fue mayor también el porcentaje de los que no dedicaban tiempo a estudiar entre los que abandonaron. Sin embargo, un $74,2 \%$ de los que prolongaron los estudios señaló haber dedicado bastante tiempo al estudio, y reconocieron que el tiempo dedicado les influyó en el rendimiento. Este tiempo dedicado al estudio influyó bastante en la finalización de estudios para los que terminaron (92,5\%), a la vez que para el 59,5\% de los que abandonaron reconocieron haber dedicado poco tiempo, y que ello influyó en su decisión de abandonar.

La realización de actividades educativas paralelas a las específicas de la titulación parece que ayuda en la carrera académica, pues los que finalizaron indicaron que habían realizado muchas actividades (74'1\%), y que ello había influido positivamente; también los que prolongan consideraron que ello les ayudó a finalizar. Sin embargo, el 76,6\% de los que abandonaron dijeron que no habían realizado ninguna actividad complementaria, y que ello tuvo poca influencia en su situación de abandono.

Sólo el 37\% de los que terminaron señaló que había utilizado bastantes estrategias a lo largo de sus estudios, y en menor proporción los que prolongaron y abandonaron. Sin embargo, tanto los que terminaron $(68,5 \%)$ como los que prolongaron (61,9\%) señalaron que el uso de técnicas de trabajo intelectual había tenido bastante influencia en su proceso formativo. El alumnado usó poco las estrategias de aprendizaje pero reconoció su importancia académica. Los tres grupos indicaron también que las técnicas usadas habían sido buenas o adecuadas, excepto un amplio porcentaje de los que abandonaron $(40,4 \%)$ que indicaron que fueron inadecuadas, pero que esto no fue lo que influyó en sus decisiones de abandonar.

El 96,3\% de los que terminaron indicaron que habían asistido siempre o regularmente a clase, aunque los otros dos grupos también lo hacían con regularidad. Sin embargo, para los que terminaron asistir a clase les ayudó a finalizar la carrera a tiempo, mientras que los que abandonaron consideraron que este hecho no estuvo nada relacionado con sus decisiones de abandonar. El alumnado que más asiste y valora las tutorías son los que prolongan. Los que abandonan son los que menos acuden, aunque las diferencias respecto a los que terminan son escasas. Tanto los que prolongan como los que terminan consideran que ello les ayudó a terminar la carrera, mientras que los que abandonan consideran en su mayoría que no asistir a tutorías no tiene por qué influir en su decisión de abandonar; sólo el 29,8\% consideró que el no asistir a tutoría pudo haber influido en el abandono.

El alumnado que termina realiza de modo regular trabajos en grupo, y consulta bastante los fondos bibliográficos, y, además, considera que ello ha teniendo mucha influencia en los logros alcanzados. Para los que prolongaron, esta dinámica fue también bastante habitual, pero no en los que abandonaron entre los que encontramos que el 63,8\% nunca o pocas veces habían realizado trabajos en grupo. Como en variables anteriores los que abandonan consideran que ello no ha tenido influencia en sus situaciones de abandono (70,2\%), opinión que contrasta con la de los que terminaron, que consideran $(90,8 \%)$ que había tenido mucha importancia en los logros alcanzados. Sin duda, este es un factor que tiene que ver con las características de las distintas titulaciones analizadas. Hay titu- 
Cabrera, L., Bethencourt, J. T., González Afonso, M. y Álvarez Pérez, P. (2006). Un estudio transversal retrospectivo sobre prolongación y abandono de estudios universitarios. RELIEVE, v. 12, n. 1, p. 105-127. http://www.uv.es/RELIEVE/v12n1/RELIEVEv12n1_1.htm

laciones con una amplia tradición en la realización de trabajos de grupo (vgr. pedagogía), y otras donde el estudio individual suele tener más peso específico (vgr. matemáticas).

Los análisis de Chi cuadrado realizados ponen de manifiesto diferencias significativas entre los tres grupos, tanto en posesión como en influencia, en todas las variables del apartado de técnicas y herramientas de trabajo intelectual, salvo en lo referido al uso de técnicas de estudio y asistencia a las tutorías.

\subsection{Características Psicoeducativas del Alumnado}

En el presente apartado presentamos los resultados relativos a la percepción de los encuestados sobre el grado de posesión de ciertas características psicoeducativas, así como la valoración hecha sobre el grado de influencia de las mismas en sus respectivas situaciones académicas. A la luz de los resultados generales obtenidos podemos dibujar el siguiente perfil de cada uno de los tres subgrupos, que podemos completar con los datos de las tablas 4 y 5 .

Tabla 4. Percepción del grado de posesión de las características psicoeducativas

\begin{tabular}{|l|c|c|c|c|}
\cline { 2 - 5 } \multicolumn{1}{c|}{} & TERMINAN & PROLONGAN & ABANDONAN & Chi $^{2}$ \\
\hline Calificaciones académicas altas & $94,4 \%$ & $75,8 \%$ & $29,8 \%$ & 0,000 \\
\hline Mapacidades para los estudios & $98,1 \%$ & $93,5 \%$ & $61,7 \%$ & 0,000 \\
\hline Conocimién hacia los estudios & $96,2 \%$ & $77,4 \%$ & $49,0 \%$ & 0,000 \\
\hline Satisfacción con la carrera elegida & $24,1 \%$ & $17,7 \%$ & $31,9 \%$ & 0,062 \\
\hline Participación e integración universidad alta & $92,6 \%$ & $79,0 \%$ & $36,2 \%$ & 0,000 \\
\hline Información y orientación recibida & $79,7 \%$ & $53,2 \%$ & $72,4 \%$ & 0,005 \\
\hline Claridad en las metas a largo plazo & $38,9 \%$ & $41,9 \%$ & $10,6 \%$ & 0,000 \\
\hline Fuerza de voluntad y constancia estudio & $85,2 \%$ & $70,9 \%$ & $63,8 \%$ & 0,040 \\
\hline Motivación de logro & $96,3 \%$ & $66,1 \%$ & $55,3 \%$ & 0,000 \\
\hline Tolerancia a la frustración & $90,7 \%$ & $83,9 \%$ & $70,2 \%$ & 0,102 \\
\hline Neces. continua experim.nuevas sensaciones & $68,6 \%$ & $69,4 \%$ & $59,6 \%$ & 0,298 \\
\hline Capac. para demorar obtención recompensas & $88,9 \%$ & $61,3 \%$ & $61,7 \%$ & 0,177 \\
\hline
\end{tabular}

Tabla 5. Valoración de influencia de las características psicoeducativas

\begin{tabular}{|c|c|c|c|c|}
\hline & TERMINAN & PROLONGAN & ABANDONAN & $\mathrm{Chi}^{2}$ \\
\hline Calificaciones académicas altas & $77,8 \%$ & $71,0 \%$ & $57,5 \%$ & 0,000 \\
\hline Capacidades para los estudios & $96,3 \%$ & $75,8 \%$ & $38,3 \%$ & 0,000 \\
\hline Motivación hacia los estudios & $94,4 \%$ & $83,9 \%$ & $68,1 \%$ & 0,005 \\
\hline Conocimientos previos & $22,3 \%$ & $37,1 \%$ & $48,9 \%$ & 0,013 \\
\hline Satisfacción con la carrera elegida & $92,6 \%$ & $75,8 \%$ & $65,9 \%$ & 0,000 \\
\hline Participación e integración universidad & $55,5 \%$ & $59,6 \%$ & $25,5 \%$ & 0,000 \\
\hline Información y orientación recibida & $37,1 \%$ & $58,1 \%$ & $36,2 \%$ & 0,019 \\
\hline Claridad en las metas a largo plazo & $87,0 \%$ & $69,3 \%$ & $68,1 \%$ & 0,324 \\
\hline Fuerza de voluntad y constancia estudio & $94,4 \%$ & $83,9 \%$ & $70,2 \%$ & 0,017 \\
\hline Motivación de logro & $81,5 \%$ & $74,2 \%$ & $63,8 \%$ & 0,415 \\
\hline Tolerancia a la frustración & $63,0 \%$ & $58,1 \%$ & $42,5 \%$ & 0,004 \\
\hline Neces. continua experim. nuevas sensaciones & $44,4 \%$ & $51,6 \%$ & $44,7 \%$ & 0,144 \\
\hline Capac. para demorar obtención recompensas & $61,2 \%$ & $59,7 \%$ & $42,5 \%$ & 0,058 \\
\hline
\end{tabular}

El comportamiento de los subgrupos en este bloque de variables fue similar al de los anteriores, con porcentajes más altos en el grupo que terminó, que fueron disminuyendo progresivamente en el que prolongó, resultando siempre inferior en el grupo que abandonó. Además, tal como reflejan los análisis del Chi cuadrado (tabla 4) se observaron 
Cabrera, L., Bethencourt, J. T., González Afonso, M. y Álvarez Pérez, P. (2006). Un estudio transversal retrospectivo sobre prolongación y abandono de estudios universitarios. RELIEVE, v. 12, n. 1, p. 105-127. http://www.uv.es/RELIEVE/v12n1/RELIEVEv12n1_1.htm

diferencias significativas entre los grupos en calificaciones académicas, capacidades para los estudios, motivación hacia los estudios, satisfacción con la carrera elegida, participación e integración en la Universidad, información y orientación recibida, claridad en las metas a largo plazo, fuerza de voluntad y constancia en el estudio y capacidad para demorar la obtención de recompensa.

Respecto a la influencia que el alumnado consideró que ha tenido este ámbito (características psicoeducativas) en sus respectivas situaciones, encontramos de nuevo la misma tendencia: los tres grupos opinaron que todas las variables analizadas tuvieron relevancia en sus situaciones académicas, siempre con porcentajes más elevados en el grupo que finalizó y menos elevados en el que abandonó. Además, sí observamos el resultado del Chi cuadrado (tabla 5) encontramos diferencias significativas en todas las variables de este apartado, excepto en claridad de las metas a largo plazo, motivación de logro, necesidad continua de experimentar nuevas sensaciones y capacidad para demorar la obtención de recompensas.

\subsection{Características bio-socio- económicas del alumnado}

Como en los apartados anteriores, quisimos primero comprobar si durante los estudios se produjeron problemas en sus vidas, bien de enfermedad, escasez de recursos u otros, para observar posteriormente si esas situaciones influyeron en la prolongación o abandono de los estudios. Los resultados obtenidos se recogen en la tabla 6 , donde se observan porcentajes casi similares de presencia de enfermedades en ellos o en familiares en los tres grupos, aunque ligeramente superior en el grupo que prolongó; de hecho, son éstos los que manifestaron $(73,1 \%)$ que esta circunstancia les influyó mucho en el retraso de los estudios. En los otros dos grupos también influyó pero en porcentajes inferiores. Por otro lado, también existe un porcentaje mayor de problemas personales y familiares, y situaciones de simultaneidad de estudios con otras actividades laborales en el grupo que prolonga. Los tres grupos consideran que estas situaciones influyen negativamente en el desarrollo de la carrera, pero mucho menos en el grupo que termina, así lo evidencia el hecho de haber terminado a pesar de haber sufrido circunstancias vitales estresantes.

Finalmente, destacar la baja presencia de situaciones vitales cambiantes o puntuales que requieren adaptación, y pocas dificultades económicas en el grupo que abandona. Sin embargo, el $100 \%$ manifestó que estas situaciones habían influido mucho en dejar o abandonar los estudios, mientras que para los que terminaron, a pesar de existir un mayor porcentaje de estas situaciones, la influencia fue poca.

Tabla 6. Valoración de influencia de las características bio-socio-económicas

\begin{tabular}{|l|c|c|c|}
\hline & TERMINAN & PROLONGAN & ABANDONAN \\
\hline Enfermedades propias o familiares & $33,3 \%$ & $41,9 \%$ & $19,1 \%$ \\
\hline Problemas personales o familiares & $37 \%$ & $54,8 \%$ & $27,7 \%$ \\
\hline Situaciones vitales que afectaron & $24,1 \%$ & $24,2 \%$ & $4,3 \%$ \\
\hline Simultaneidad de estudios otras actividades & $42,6 \%$ & $59,7 \%$ & $31,9 \%$ \\
\hline Dificultades económicas & $20,4 \%$ & $27,4 \%$ & $6,4 \%$ \\
\hline
\end{tabular}

\subsection{Características de la carrera: plan de estudio/ infraestructura/ (recursos materiales, personales y organizativos)}

Con el rótulo "características de la carrera" pretendimos indagar sobre la valoración del alumnado de la titulación, es decir, características del plan de estudios, infraestructura de las instalaciones de la facultad o centro, otros recursos puestos en práctica para el desarrollo o aprendizaje de las materias, funcionamiento del centro, etc. En la tabla 7 
Cabrera, L., Bethencourt, J. T., González Afonso, M. y Álvarez Pérez, P. (2006). Un estudio transversal retrospectivo sobre prolongación y abandono de estudios universitarios. RELIEVE, v. 12, n. 1, p. 105-127. http://www.uv.es/RELIEVE/v12n1/RELIEVEv12n1_1.htm

podemos observar las variables analizadas y la valoración dada a cada una de ellas.

Tabla 7. Percepción del grado de posesión de las características de la carrera

\begin{tabular}{|l|c|c|c|c|}
\cline { 2 - 5 } \multicolumn{1}{c|}{} & TERMINAN & PROLONGAN & ABANDONAN & Chi $^{2}$ \\
\hline Adecuación del contenido de las asignaturas & $79,6 \%$ & $77,4 \%$ & $61,7 \%$ & 0,023 \\
\hline Formación práctica recibida & $48,2 \%$ & $29,1 \%$ & $38,3 \%$ & 0,001 \\
\hline Servicios & $51,9 \%$ & $53,5 \%$ & $38,3 \%$ & 0,573 \\
\hline Utilidad de contenidos para el desempeño & $57,5 \%$ & $38,7 \%$ & $36,2 \%$ & 0,316 \\
\hline Nivel de exigencia & $63,0 \%$ & $61,3 \%$ & $68,1 \%$ & 0,003 \\
\hline Masificación & $46,3 \%$ & $71,0 \%$ & $68,1 \%$ & 0,024 \\
\hline Ambiente académico adecuado & $85,2 \%$ & $66,1 \%$ & $63,8 \%$ & 0,244 \\
\hline Relación con los compañeros & $92,6 \%$ & $88,7 \%$ & $74,4 \%$ & 0,137 \\
\hline
\end{tabular}

En este apartado se apreciaron algunas diferencias altamente significativas entre los tres grupos respecto a la valoración de las variables analizadas (Tabla 7).

Respecto al nivel de influencia, encontramos que en el grupo que terminó sus estudios lo que más les influyó fue: la formación práctica recibida $(81,4 \%)$, el contenido de las asignaturas $(79,7 \%)$, la relación con los compañeros (77,8\%), el ambiente académico $(70,4 \%)$ y la utilidad de los contenidos para el ejercicio de la profesión (68,6\%). En el grupo que prolonga se observan porcentajes similares, sin embargo, los que abandonaron consideraron que les influyó para dejar la titulación los contenidos de las asignaturas (57,5\%), el nivel de exigencia (49\%) y la utilidad de los contenidos (48\%), mientras que las otras variables tienen influencia en porcentajes bastante inferiores. En todas las variables el nivel de influencia fue estadísticamente significativo, excepto en la variable utilidad de los contenidos.

\subsection{Características del profesorado}

Las características del profesorado, sus cualidades profesionales y el desempeño de la docencia se han identificado siempre como factores influyentes en el rendimiento del alumnado. En este sentido, consideramos que si el rendimiento es un determinante del abandono, indirectamente también podría serlo el profesorado. Por ello, preguntamos al alumnado sobre su percepción sobre estos aspectos.

Los resultados muestran una valoración muy positiva del profesorado por parte de todo el alumnado. Además, se constataron diferencias significativas en la influencia de todas las variables en los tres grupos, es decir, se mostró la alta influencia del rol del profesorado en el alumnado para que terminara, y la baja influencia de éste en sus decisiones de abandonar. Los tres grupos consideraron que las actitudes del profesorado hacia la materia que impartía y hacia el alumnado fueron positivas; el nivel de exigencia era alto y eran competentes profesionalmente; las interacciones que se daban día a día entre el profesorado y el alumnado eran adecuadas o buenas y la metodología didáctica y el sistema de evaluación utilizado en sus asignaturas fueron también adecuados. Dentro de este perfil, destacar solamente la percepción por parte del grupo que abandonó de que la calidad de las interacciones entre profesorado y alumnado no era tan buena, con diferencias estadísticamente significativas. 
Cabrera, L., Bethencourt, J. T., González Afonso, M. y Álvarez Pérez, P. (2006). Un estudio transversal retrospectivo sobre prolongación y abandono de estudios universitarios. RELIEVE, v. 12, n. 1, p. 105-127. http://www.uv.es/RELIEVE/v12n1/RELIEVEv12n1_1.htm

Tabla 8. Percepción del grado de posesión de las características del profesorado

\begin{tabular}{|l|c|c|c|c|}
\cline { 2 - 5 } \multicolumn{1}{c|}{} & TERMINAN & PROLONGAN & ABANDONAN & Chi $^{2}$ \\
\hline Actitud del profesorado hacia la enseñanza & $92,6 \%$ & $75,8 \%$ & $70,2 \%$ & 0,034 \\
\hline Nivel de exigencia del profesorado & $77,8 \%$ & $72,6 \%$ & $72,4 \%$ & 0,552 \\
\hline Competencia profesional del profesorado & $66,7 \%$ & $66,1 \%$ & $63,8 \%$ & 0,332 \\
\hline Calidad interacción profesor-alumno & $74,1 \%$ & $66,2 \%$ & $42,6 \%$ & 0,008 \\
\hline Metodología docente & $57,5 \%$ & $61,3 \%$ & $66,0 \%$ & 0,124 \\
\hline Tipo de evaluación & $77,8 \%$ & $69,4 \%$ & $68,9 \%$ & 0,435 \\
\hline
\end{tabular}

Tabla 9. Valoración del grado de influencia de las características del profesorado

\begin{tabular}{|l|c|c|c|c|}
\cline { 2 - 5 } \multicolumn{1}{c|}{} & TERMINAN & PROLONGAN & ABANDONAN & Chi $^{2}$ \\
\hline Actitud del profesorado hacia la enseñanza & $75,9 \%$ & $79,1 \%$ & $36,2 \%$ & 0,000 \\
\hline Nivel de exigencia del profesorado & $29,6 \%$ & $59,6 \%$ & $36,1 \%$ & 0,000 \\
\hline Competencia profesional del profesorado & $44,4 \%$ & $56,4 \%$ & $19,2 \%$ & 0,000 \\
\hline Calidad interacción profesor-alumno & $64,8 \%$ & $69,3 \%$ & $25,5 \%$ & 0,000 \\
\hline Metodología docente & $61,1 \%$ & $74,2 \%$ & $29,8 \%$ & 0,000 \\
\hline Tipo de evaluación & $48,2 \%$ & $66,1 \%$ & $14,9 \%$ & 0,000 \\
\hline
\end{tabular}

En cuanto al nivel de influencia de cada una de estas variables encontramos que la actitud del profesorado influyó mucho en el alumnado para que terminara los estudios, pero no influyó en la decisión de abandonar. Sobre el nivel de exigencia, a quienes más influyó en sus situaciones fue a los que prolongaron, concretamente a un 59,64\%; para los que terminaron y abandonaron esta variable tuvo una influencia bastante inferior. Igualmente baja parece ser la influencia de la competencia profesional del profesorado, ejerciendo muy poca influencia en el grupo que abandonó y un poco más (alrededor del $50 \%$ ) en los otros dos grupos. Esta tendencia grupal es similar en la valoración de la interacción profesorado-alumnado; el grupo que terminó y el que prolongó consideró que ello influyó bastante es su situación académica, sin embargo, el grupo que abandonó consideró que ello no influyó o influyó poco en su decisión.

A juicio de los que terminaron y prolongaron la metodología docente tuvo bastante o mucha influencia en el desarrollo de la carrera académica, sin embargo, para los que abandonaron este hecho influyó poco o nada en su decisión. Finalmente, para el 48,2\% de los que terminaron y para el $66,1 \%$ de los que prolongaron, el tipo de evaluación influyó bastante o mucho en los logros alcanzados, pero muy poco para los que abandonaron.

\section{Discusión y Conclusiones}

La situación académica de los encuestados en el momento en el que se recogieron los datos confirmó la tendencia marcada por otros estudios previos, donde los porcentajes de abandono se sitúan alrededor del 25\%. En nuestro caso se observa una impresionante variación por titulaciones, circunstancia también reflejada en otros estudios similares. Son escasos los trabajos realizados sobre el análisis de las variables diferenciales intervinientes, sin embargo, los argumentos de mayor consistencia hacen referencia a los conocimientos previos y al acceso restringido. Los escasos conocimientos previos afectarían más a las titulaciones técnicas, de ahí el presumible aumento de abandonos en estas titulaciones. Sin embargo, no se puede atribuir toda la responsabilidad al alumnado, máxime cuando se ha demostrado que muchos de los alumnos que abandonan sus titulaciones no tienen fracaso académico. Respecto al acceso restringido, parece que el alumno que lucha por entrar en una titulación 
de difícil acceso, lo hace porque tiene una fuerte motivación hacia esos estudios, y ésta le ayuda a esforzarse y demorar la recompensa hasta el final, independientemente de la dificultad de los mismos. En España podemos observar este hecho en el alumnado de medicina donde se registran muy pocos casos de abandono. En nuestro estudio la única titulación con acceso restringido es trabajo social y, de hecho, se registra un porcentaje muy bajo de abandono. Son hipótesis, sin embargo, que exigen mayor confirmación.

En relación a las variables influyentes, los resultados nos sugieren que tanto las variables del alumnado como las variables del contexto son determinantes de dicho fenómeno problemático, pero las primeras son más influyentes que las segundas. Dentro de las variables del alumnado las más influyentes resultaron ser las características psicológicas y las estrategias y actividades de estudio.

En cuanto a las estrategias y herramientas de trabajo intelectual, los tres grupos (mayor porcentaje entre los que terminan) consideran que el uso de técnicas adecuadas es determinante en el desarrollo de sus estudios con éxito, aunque luego no hagan uso de ellas. Otras variables relevantes en este conjunto fueron la asistencia a clase y a tutorías, y la realización de actividades complementarias. Para los que finalizan los estudios estas prácticas contribuyeron y les facilitaron terminar con éxito, pero los que terminan consideran que el poco uso o escasa asistencia no fue lo que influyó en sus decisiones para abandonar. De modo sistemático encontramos que los tres grupos valoran de una forma muy similar los factores favorecedores para culminar la carrera, sin embargo, los que no lo hacen, no reconocen el peso específico de cada una de ellos en sus decisiones de abandonar; según nuestra hipótesis, en el abandono son muchas las variables intervinientes, convirtiendo el hecho en un fenómeno de naturaleza multicausal, por lo que a los implicados les resulta difícil reconocer la influencia de cada una de ellas de forma aislada. La influencia de las estrategias de estudios ha sido identificada en otros estudios, sobre todo en aquellos que han mostrado las exitosas experiencias de instrucción en estrategias de aprendizaje puestas en práctica con la finalidad de incrementar las tasas de retención del alumnado.

Las características psicoeducativas se erigen en el factor más relevante a la hora de explicar el abandono. En nuestro estudio constatamos diferencias significativas entre los tres grupos. Los que terminan, frente a los que abandonan, manifiestan un mayor nivel de satisfacción con los distintos aspectos que han formado parte de su proceso formativo, tales como, motivación, calificaciones académicas, satisfacción con los estudios; los que abandonan manifiestan tener menos motivación, bajas calificaciones y consideran que no poseen las capacidades adecuadas para cursar estudios universitarios. Sin embargo, resulta interesante observar como el alumnado que abandona estudios de alto nivel de exigencia (matemáticas) atribuye esta circunstancia a la titulación, a su dificultad y a la desmotivación provocada por la falta de recompensas; y el que abandona estudios de bajo nivel de dificultad (trabajo social) lo atribuye a motivos personales y económicos. Este tipo de consideraciones debería constituir un aspecto a valorar a la hora de llevar a cabo la orientación y la planificación universitaria; de hecho, los tres grupos manifiestan haber recibido escasa información y orientación sobre itinerarios académicos en la enseñanza secundaria. Estas circunstancias propician la toma de decisiones del alumnado guiada por inercias, sin una conciencia clara de las capacidades necesarias para concluir con éxito los estudios elegidos, ni de lo que supone ser estudiante universitario. Informar y orientar al alumnado, así como valorar sus potencialidades, constituye una acción preventiva que permite hacer elecciones adecuadas y evitar situaciones como las que hemos visto en este trabajo, 
tales como las de abandonar o prolongar los años de estudio.

En contra de nuestras hipótesis iniciales, se constata una escasa incidencia en el abandono de problemas particulares relacionados con la salud, la economía u otras situaciones de estrés personal. Estas circunstancias se dan por igual en los tres grupos, sin embargo a quienes menos afectan es al grupo que termina. Este hecho nos obliga a hacer una relación con las variables psicológicas del alumnado, donde entendemos que el alumnado con mayor resistencia a situaciones difíciles tiene más probabilidades de terminar con éxito los estudios, porque sabe manejar mejor las situaciones de estrés.

Las variables académicas, tanto de la titulación como del profesorado, tienen poca relevancia en las decisiones del alumnado. Destacar, como en el capítulo de estrategias de estudio, que el alumnado que termina considera que el profesorado influyó mucho en ello. A pesar de que el conjunto del alumnado hace una valoración positiva del profesorado, el que abandona no se benefició de la ayuda que éste le podía prestar, bien por escasa empatía, o porque no descubrió esa posibilidad, pues no reconocen la influencia del profesorado en sus decisiones.

Un aspecto a resaltar es el hecho de que la mayoría de los estudiantes que abandonan no dejan la Universidad, sino que siguen estudiando en otra titulación, de lo que se deduce cierta insatisfacción con la titulación y no con la Universidad o los estudios universitarios. Otras cuestiones son las razones que determinan o provocan la insatisfacción con la titulación, que según los datos resultantes serían atribuibles a la saturación del mercado laboral, las pocas facilidades por parte de las respectivas facultades, imagen errónea de la titulación elegida antes de iniciarla, etc. Por otro lado, los motivos de elección de la carrera tampoco parece ser una variable determinante en la posterior situación académica del alumnado, dado que en los tres coincide como motivo de elección preferente el gusto por los estudios, aunque se observa una tendencia de autoexclusión del propio alumnado de aquellas titulaciones para las que no se siente capacitado, por miedo al fracaso.

Respecto a la metodología utilizada, se cumplieron todas las expectativas al permitirnos reconstruir el comportamiento del alumnado frente a los estudios que inicia en un momento temporal. Sucesivos estudios transversales de estas características, en distintos momentos de tiempo, nos permitirán abordar en el futuro el estudio del abandono a partir de series temporales. Las series temporales constituyen una de las técnicas más habituales para prever los fenómenos de cualquier naturaleza, utilizándose con el propósito de describir la "historia" de una determinada variable o variables. Este estudio, una vez identificadas las variables relacionadas o predictivas, nos permitirá hacer análisis de tendencias o variación; es decir, construir un modelo que permita, a través de técnicas de series temporales (Ejm. Modelo ARIMA, enfoque moderno de series temporales para hacer predicciones a corto plazo), analizar el transcurso del fenómeno del abandono.

\section{Referencias bibliográficas}

Airbar, C.; Rabanaque, M.J.; AlvarezDardet, C.; Nolasco, A.; Moncho, J.; Gascón, E. (1999). Evolución de los diseños epidemiológicos de la investigación clínica en España (1975-1994). Revista española de salud pública, 73, 4, 445-453.

Albert, C. y Toharia, L. (2000). El abandono o la persistencia en los estudios universitarios. Papeles de Economía Española, 86, 192-212.

Bean, J.P. (1990). Understanding why students stay or leave. En D. Hossler, J.P. Bean, and assoc. (Eds.), The Strategic Management of College Enrollments, San Francisco: Jossey-Bass.

Callejo, J. (2001). A Cohorty study on UNED students: an aproximation to dropout analysis. Revista Iberoamericana de Educación a Distancia, 4, 2, 33-69. 
Colimón, K. (1990). Fundamentos de epidemiología. Madrid: Díaz de Santos, S.A.

Corominas, E. (2001). La transición a los estudios universitarios. Abandono o cambio en el primer año de universidad. Revista de Investigación Educativa, 19 (1), 127-151.

Escandell, O. y Marrero, G. (1999). El abandono de los estudiantes en la universidad de Las Palmas de Gran Canaria: sus causas, propuestas de estrategias para su disminución. Las Palmas de Gran Canaria: La Caja de Canarias.

Escandell, O; Marrero, G. y Castro, J.J. (2002). El abandono universitario: la opinión de los estudiantes de la ULPGC. Evaluación e Intervención Psicoeducativa, 1, 8-9, 305-338.

Feldman, R. S. (2005). Improving the first year of college: Research and practice. Mahwah, NJ: LEA (Lawrence Erlbaum Associates).

González, M., Alvarez, P., Cabrera, L. y Bethencourt, J.T. (2004). Los problemas de transición y el abandono de los estudios universitarios. Conferencia Internacional de Orientación, Inclusión Social y Desarrollo de la Carrera. Universidad A Coruña.

González de Rivera y Revuelta, J.L.; Rodríguez Pulido, F.; y Sierra López, A. (1993). El método epidemiológico en salud mental. Barcelona, Ediciones científicas y técnicas, D.L.

Keeves, J.P. (Ed.) (1997). Educational research, methodology and measurement. An international handbook. Nueva York: Pergamon Press.

Kirton, M. J. (2000). Transitional factors influencing the academic persistence of first semester undergraduate freshmen. Dissertation Abstracts International Section A: $\mathrm{Hu}-$ manities and Social Sciences, 61, 2-A, 522.

Landry, C. C. (2003). Self-efficacy, motivation and outcome expectation correlates of college students' intention certainty. Dissertation Abstracts International Section A: Humanities and Social Sciences, 64, 3-A, 825.

Last, L. y Fulbrook, P. (2003). Why do student nurses leave? Suggestions from a Del- phi study. Nurse Education Today, 23, 6, 449-458.

Latiesa, M. (1992). La deserción universitaria. Madrid: CIS.

Levy-Garboua, L. (1986). Selección e ineficacia en la enseñanza superior. En M. Latiesa (Comp.). Demanda de educación superior y rendimiento académico en la universidad. Madrid: MEC.

Lipka, S. (2004). Dropout rate is worse in U.S., Report Says. Chronicle of Higher Education, 51, 5, 38-53.

López Rupérez, F. (2004). Calidad de la educación. Por dónde va el mundo y por dónde va España. Ponencia del Curso de Verano de la Universidad Internacional Menéndez Pelayo. Temas de la España actual. 1-3 Septiembre.

López Rupérez, F. (2005). Los problemas del abandono del sistema reglado. Benchmarks europeos y políticas sociales. Comunidad autónoma de Madrid.

Masjoan, J.M. (1989). Escala de valores instrumentales, autoestima y permanencia en el sistema educativo. Sociología de la Educación, 11, 169-203.

Mateo, J. (2004). La investigación ex postfacto. En Bisquerra (Coord.) Metodología de la investigación educativa. Madrid: La Muralla.

Orazem, V. (2000). Understanding why they stay and why they leave: A grounded theory investigation of undecided students at a rural grant institution. Dissertation Abstracts International Section A: Humanities and Social Sciences, 61, 6-A, 2214.

Orfield, G. (2004). Dropout in America: Confronting the graduation rate crisis. Harvard Education Press.

Root, S.; Rudawski, A; Taylor, M. y Rochon, R. (2003). Attrition of Hmong Students in Teacher Education Programs. Bilingual Research Journal, 27, 1, 137-148.

Ryan, M.P. y Glenn, P.A. (2003). Increasing one-year retention rates by focusing on academic competence: An empirical odyssey. Journal of College Student Retention, 4, 3, 297-324. 
Salthouse, T. y Schoeder, D. (2004). Estimating retest effects in longitudinal assessment of cognitive functioning in adults between 18 and 60 years of age. Developmental Psychology, 40, 5, 813-822.

Sinclair, H. and Dale, T. (2000). The effect of student tuition fees on the diversity of intake within a Scottish new university. Paper presented at British Educational Research Association Annual Conference, 7-9 September, 2000, Cardiff University.

Tinto, V. (1975). Dropout from higher education: A theoretical synthesis of recent research. Review of Educational Research, 45, 89-125.

Tinto, V. (1993). Leaving college: rethinking the causes and cures of student attrition. Chicago: University of Chicago Press.

Thomas, L. (2002). Student retention in higher education: the role of institutional habitus. Journal Education Policy. 17, 4, 423-442.

Van Ours, J.C. y Ridder, G. (2003). Fast track or failure: a study of the graduation and dropout rates of Ph.D. students in economics. Economics of Education Review, 22 (2), 157-166.

UNESCO (2004). World higher education database. Consultado el 24 de marzo de 2006 http://www.unesco.org/iau/directories/index .html

Wasserman, K. N. (2001): Psychological and development differences between students who withdraw from college for personalpsychological reasons and continuing students. Dissertation Abstracts International Section A: Humanities and Social Sciences, 62, 3-A, 915.

Zubieta, J.C. y Susinos, F. (1986). Desigualdad de formación y rendimiento académico en las escuelas universitarias. En M. Latiesa (Comp.). Demanda de educación superior y rendimiento académico en la universidad. Madrid: MEC

\section{NOTAS}

[1]La cohorte se refiere a la población o muestra objeto de estudio. No confundir con Estudios de cohorte en el que la cohorte la constituye un grupo con un factor de riesgo, que se compara con otro grupo sin el factor de riesgo.

[2] Los porcentajes, en todas las tablas, indican el rango positivo de la escala de valoración, es decir, la cantidad de personas que indican mucho o bastante. 


\section{ABOUT THE AUTHORS / SOBRE LOS AUTORES}

Lidia Cabrera Pérez (dcabrera@ull.es): es profesora titular del área Métodos de Investigación y Diagnóstico en Educación (MIDE); actualmente imparte materias de diagnóstico y evaluación en la titulación de psicopedagogía, y sus líneas de investigación están relacionadas con la orientación en la universidad y la evaluación e intervención socioeducativa. Su dirección postal es Departamento de Didáctica e Investigación Educativa. Facultad de Educación. Campus Central. Universidad de La Laguna. 38204-La Laguna. Tenerife.

José Tomás Bethencourt Benítez (jbethen@ull.es): profesor titular del área de Psicología Evolutiva y de la Educación; imparte materias de orientación profesional y estimulación intelectual en la titulación de psicopedagogía y psicología respectivamente, y sus líneas de investigación están relacionadas con la psicología escolar.

Miriam González Afonso (mcglez@ull.es): es profesora ayudante del área de Didáctica y Organización Escolar, e imparte materias orientación y tutoría en diferentes titulaciones de Psicopedagogía, Maestro Especialista en Infantil y Musical. Sus áreas de trabajo están relacionadas con formación del profesorado universitario y la orientación la tutoría en la enseñanza superior.

Pedro Álvarez Pérez (palvarez@ull.es): profesor titular de Orientación Profesional del área de Didáctica y Organización Escolar. Imparte materias de orientación y tutoría en las titulaciones de Psicopedagogía, Curso de Cualificación Pedagógica y Títulos de Maestro Especialista. Sus áreas de trabajo están centradas en el ámbito de la orientación sociolaboral, orientación profesional a colectivos de riesgo y orientación y tutoría universitaria. 
Cabrera, L., Bethencourt, J. T., González Afonso, M. y Álvarez Pérez, P. (2006). Un estudio transversal retrospectivo sobre prolongación y abandono de estudios universitarios. RELIEVE, v. 12, n. 1, p. 105-127.

http://www.uv.es/RELIEVE/v12n1/RELIEVEv12n1_1.htm

\section{ARTICLE RECORD / FICHA DEL ARTÍCULO}

\begin{tabular}{|c|c|}
\hline $\begin{array}{l}\text { Reference / } \\
\text { Referencia }\end{array}$ & $\begin{array}{l}\text { Cabrera, Lidia, Bethencourt, José Tomás, González Afonso, Miriam y Álvarez Pérez, Pedro (2006). Un } \\
\text { estudio transversal retrospectivo sobre prolongación y abandono de estudios universitarios. } \frac{\text { RELIEVE, } v \text {. }}{\text { 12, n. 1. http://www.uv.es/RELIEVE/v12n1/RELIEVEv12n1_1.htm. Consultado en (poner fecha). }}\end{array}$ \\
\hline Title / Título & $\begin{array}{l}\text { Un estudio transversal retrospectivo sobre prolongación y abandono de estudios universitarios. [A retro- } \\
\text { spective transversal study of enlargement and college dropout] }\end{array}$ \\
\hline $\begin{array}{l}\text { Authors / } \\
\text { Autores }\end{array}$ & Lidia Cabrera Pérez, José Tomás Bethencourt Benítez, Miriam González Afonso, y Pedro Álvarez Pérez \\
\hline $\begin{array}{l}\text { Review / } \\
\text { Revista }\end{array}$ & Revista ELectrónica de Investigación y EValuación Educativa (RELIEVE), v. 12, n. 1 \\
\hline ISSN & $1134-4032$ \\
\hline $\begin{array}{l}\text { Publication } \\
\text { date / } \\
\text { Fecha de } \\
\text { publicación }\end{array}$ & $\begin{array}{l}2006 \text { (Reception Date: } 2005 \text { Nov. 22; Approval Date: } 2006 \text { April 23; Publication Date: } 2006 \text { May } \\
\text { 25) }\end{array}$ \\
\hline $\begin{array}{l}\text { Abstract / } \\
\text { Resumen }\end{array}$ & $\begin{array}{l}\text { At present leaving and prolonging studies constitutes a serious problem at the university level. This re- } \\
\text { port presents the results of a retrospective transversal study which purpose was to identify risk factors } \\
\text { related with dropout. The methodology design used can be for help in the building of predictive models } \\
\text { about dropout. } \\
\text { El abandono y la prolongación de los estudios constituyen en la actualidad un importante problema en } \\
\text { la Universidad. En el presente informe se presentan los resultados de un estudio transversal retrospectivo } \\
\text { que tenía por objetivo identificar los factores relacionados con la prolongación y el abandono de estu- } \\
\text { dios. Para su consecución, se ha utilizado un diseño metodológico que consideramos permite construir } \\
\text { modelos predictivos y de tendencia en el futuro. }\end{array}$ \\
\hline $\begin{array}{l}\text { Keywords } \\
\text { Descriptores }\end{array}$ & $\begin{array}{l}\text { Dropout studies; university orientation; disability learning; retrospective study; transversal study } \\
\text { Abandono de estudios; orientación universitaria; dificultades de aprendizaje; estudios retrospectivos; } \\
\text { estudios transversales }\end{array}$ \\
\hline $\begin{array}{l}\text { Institution / } \\
\text { Institución }\end{array}$ & Universidad de La Laguna (Spain) \\
\hline $\begin{array}{l}\text { Publication } \\
\text { site / } \\
\text { Dirección }\end{array}$ & http://www.uv.es/RELIEVE \\
\hline $\begin{array}{l}\text { Language / } \\
\text { Idioma }\end{array}$ & Spanish (Title, abstract and keywords in English ) \\
\hline
\end{tabular}

\section{Revista ELectrónica de Investigación y EValuación Educativa (RELIEVE)}

[ ISSN: 1134-4032 ]

(C) Copyright, RELIEVE. Reproduction and distribution of this articles it is authorized if the content is no modified and their origin is indicated (RELIEVE Journal, volume, number and electronic address of the document).

(C) Copyright, RELIEVE. Se autoriza la reproducción y distribución de este artículo siempre que no se modifique el contenido y se indique su origen (RELIEVE, volumen, número y dirección electrónica del documento). 